\title{
Spectral Analysis Methods for Poisson Sampled Measurements
}

\author{
Reinder Banning
}

\begin{abstract}
The velocity measurements for turbulent flow regimes obtained with laser doppler anemometry are not only affected by random noise but are also unevenly spaced in time. The usual spectral estimators rely on evenly spaced data points. It would appear then that the measurement data requires adjustment before it can be passed on to these estimators. In this paper, both an analysis method with a novel adjustment scheme as well as an analysis method which does not rely on the use of adjustment schemes, are presented.
\end{abstract}

Index Terms - Discrete Fourier transforms, Kalman filtering, Poisson processes, sampled data systems, spectral analysis, stochastic systems.

\section{INTRODUCTION}

$\mathbf{T}$ HE velocity of a fluid particle travelling along a streamline in a homogeneous isotropic turbulent flow may be measured using the digital laser Doppler anemometry (LDA) measurement technique. Unfortunately, LDA measurements are affected by stochastic disturbances arising from laserbeam imperfections and the use of electronic equipment, which will find their way into the spectral estimation results. In addition, these velocity measurements are sampled according to a Poisson sampling scheme culminating in an unevenly spaced time series.

The best known and numerically most efficient spectral estimation tools rely upon the fast Fourier transform (FFT) algorithm, which demands an evenly sampled time series. Consequently, unevenly spaced measurement data can not be processed by any of these tools. Traditionally, data adjustment schemes are used for getting around this problem by creating evenly spaced data points.

In this paper, a spectral analysis method is presented which exploits a novel adjustment scheme relying upon the Kalman Filter [1] used for disturbance attenuation. Furthermore, a second analysis method is suggested which circumvents the need for data adjustment altogether, and which interfaces directly with the existing spectral estimation tools.

\section{LAGRANGIAN MODELING}

Consider a dynamic model in the form of a linear stochastic differential equation for velocity $\{v(t)\}$ of a generic fluid particle travelling along a stream-line in a homogeneous

Manuscript received June 3, 1996.

The author is with the Faculty of Applied Physics, Signal Processing Group, Delft University of Technology, Delft 2628 CJ, The Netherlands (e-mail: banning@tn.tudelft.nl).

Publisher Item Identifier S 0018-9456(97)06476-0. isotropic turbulent flow [2]

$$
\frac{\mathrm{d} v(t)}{\mathrm{d} t}=-\mathbf{a} v(t)+\mathbf{b} d(t) ; \quad v(0)=v_{0}
$$

for time-invariant coefficients a and $\mathbf{b}$. Furthermore, parameter $\mathbf{a}$ of the drift term $\mathbf{- a} v(t)$ is assumed to be positive while parameter $\mathbf{b}$ is allowed to take on any real value. The term $\mathbf{b} d(t)$ depends upon diffusion process $\{d(t)\}$ which is, by assumption, a stationary (i.e., with time invariant mean and standard deviation as well as an autocovariance $\mathcal{R}_{d}(t+\tau, t)$ depending upon $\tau$ only) Gaussian white (i.e., independent in time and with constant mean and standard deviation) noise process with mean $\mu$ and standard deviation $\sigma_{n}$. Because the velocity of a typical fluid particle at initial time $t=0$ is unknown, we represent $v(0)=v_{0}$ by a Gaussian stochastic variable with mean $v_{0}$ and standard deviation $\sigma_{v}$ which is independent from $\{d(t)\}$.

Traditionally, the diffusion process driving this model is a Gaussian white noise process with a flat power spectrum. Consequently, the magnitude Bode plot of the particle velocity's power spectral density function or power spectrum, is displaying a single break-frequency, corresponding with one change in rate of roll-off. However, from theoretical considerations confirmed by experimental results, [3], more than one change in rate of roll-off is known to occur. This means that the power spectrum of $\{v(t)\}$ contains at least one break-frequency. In view of these considerations we propose to represent the diffusion process not by a stationary Gaussian white noise process but by a stationary Gaussian colored noise process. Such a process is created by passing stationary Gaussian white noise through a linear time-invariant dynamic system as represented by the transfer function

$$
\mathbf{H}(s)=\frac{\mathbf{h}_{m-1} s^{m-1}+\cdots+\mathbf{h}_{1} s+\mathbf{h}_{0}}{s^{n-1}+\mathbf{g}_{n-2} s^{n-2}+\cdots+\mathbf{g}_{1} s+\mathbf{g}_{0}}
$$

with $m<n$, and $n$ asymptotically stable poles. An overall state-space model for the isotropic homogeneous turbulent velocity field known as the extended stationary Langevin model (ESLM) may then be obtained as [('A', B', C') denotes a state space realization of $\mathbf{H}(s)$ ]

$$
\left\{\begin{aligned}
\frac{d}{d t}\left(\begin{array}{l}
v(t) \\
z(t)
\end{array}\right) & =\left(\begin{array}{l|l}
\mathbf{- a} & \mathbf{b} \mathbf{C}^{\prime} \\
\hline \mathbf{\Phi} & \mathbf{A}^{\prime}
\end{array}\right)\left(\begin{array}{l}
v(t) \\
z(t)
\end{array}\right)+\left(\frac{0}{\mathbf{B}^{\prime}}\right) h(t) \\
& =\mathbf{A} x(t)+\mathbf{B} h(t) \\
v(t) & =(1 \mid \mathbf{0})\left[v(t) z(t)^{T}\right]^{T}=\mathbf{C} x(t)
\end{aligned}\right.
$$


for initial state $x\left(t_{0}\right)=x_{0}$ a Gaussian stochastic variable and driving process $\{h(t)\}$ a Gaussian white noise process such that

$$
\begin{gathered}
\mathbf{E}\{h(t)\}=\mu \\
\mathcal{R}_{h}(t+\tau, t)=\sigma_{h}^{2} \delta(\tau) \\
\mathbf{E}\left\{x_{0}\right\}=-\mathbf{A}^{-1} \mathbf{B} \mu \\
\mathbf{E}\left\{[h(t)-\mu]\left[x_{0}+\mathbf{A}^{-1} \mathbf{B} \mu\right]^{T}\right\}=0 \\
\mathbf{0}=\mathbf{A} \mathcal{R}_{x}(0,0)+\mathcal{R}_{x}(0,0) \mathbf{A}^{T}+\sigma_{h}^{2} \mathbf{B B}^{T}
\end{gathered}
$$

The continuous-time power spectral density function $\mathcal{S}_{v}(\omega)$ of stationary process $\{v(t)\}$ may be expressed in terms of matrix triplet $(\mathbf{A}, \mathbf{B}, \mathbf{C})$ as

$$
\mathcal{S}_{v \omega}=\left|\mathbf{C}[\mathbf{j} \omega \mathbf{I}-\mathbf{A}]^{-\mathbf{1}} \mathbf{B}\right|^{2} \sigma_{h}^{2} .
$$

\section{Modeling MeAsurements}

The LDA measurement technique is a discrete-time measurement technique with which the velocity of a fluid particle travelling along a stream-line in a homogeneous isotropic turbulent flow can be registered. Unfortunately, these velocity measurements are affected by disturbances, which for reasons of modeling convenience, are represented by an additive Gaussian white noise process. The digital observation equation for the ESLM is

$$
v^{o b s}\left(t_{k}\right)=v\left(t_{k}\right)+n\left(t_{k}\right)
$$

with $\left\{n\left(t_{k}\right)\right\}$ a zero mean Gaussian white noise process with standard deviation $\sigma_{n}$. There is no reason to suspect an effect from the LDA measurement technique on the velocity field, implying that $\left\{n\left(t_{k}\right)\right\},\{h(t)\}$, and $x_{0}$ may be assumed to be mutually independent.

When measuring the velocity of a turbulent flow field using LDA, the simultaneously recorded sampling instants prove to be unevenly spaced. In point of fact, in a series of LDA sampling instants the number of sampling instants per distinct time interval is Poisson distributed while the sampling intervals resemble realizations of an exponential white noise process. The propagation equation for the so-called Poisson sampling process $\left\{t_{k}\right\}$ is [4]

$$
t_{k+1}=t_{k}+\Delta t_{k} ; \quad t_{0}=0
$$

for $\left\{\Delta t_{k}\right\}$ an exponential white noise process with mean $\Delta t$, known as the sampling-interval process; $\left\{\Delta t_{k}\right\},\{h(t)\},\left\{n\left(t_{k}\right)\right\}$, and $x_{0}$ are assumed to be mutually independent.

Assume for the sake of argument that a Poisson distributed sequence of sampling instants is known in advance and let the ESLM describe some turbulent velocity field. Above observation equation relates observation process $\left\{v^{o b s}\left(t_{k}\right)\right\}$ to the output process and hence to the state process of the ESLM. Consequently, it suffices to know the system's state process at the sampling instant in order to know the observation process. Using the closed form expression for solution $\{x(t)\}$ of the
ESLM, we find

$$
x\left(t_{k+1}\right)=e^{\mathbf{A} \Delta t_{k}} x\left(t_{k}\right)+\int_{t_{k}}^{t_{k+1}} e^{A\left(t_{k+1}-u\right)} \mathbf{B} h(u) d u .
$$

The integral in this relation yields a discrete-time Gaussian white noise process, denoted by $g\left(t_{k+1}, t_{k}\right)$, with a mean and a variance determined as

$$
\begin{aligned}
\mathbf{E}\left\{g\left(t_{k+1}, t_{k}\right)\right\} & =\left\{\int_{0}^{\Delta t_{k}} e^{\mathbf{A} u} \mathbf{B} d u\right\} \mu \\
\mathcal{R}_{g}\left(t_{k}, t_{k}\right) & =\sigma_{h}^{2} \int_{0}^{\Delta t_{k}} e^{\mathbf{A} w} \mathbf{B B}^{T} e^{\mathbf{A}^{T} w} d w
\end{aligned}
$$

because the driving process of the ESLM is a (Gaussian) white noise process. After introducing notation $\Phi\left(\Delta t_{k}\right)$ for the exponential matrix of $\mathbf{A} \Delta t_{k}$, we proceed by combining above dynamic relation with the ESLM's observation equation for the purpose of obtaining a dynamic model in the discretetime domain for the Poisson sampled measurements of fluid particle velocity $\{v(t)\}$. The resulting model is of the form [equivalent discrete-time system (EDS)]

$$
\left\{\begin{array}{l}
x\left(t_{k+1}\right)=\Phi\left(\Delta t_{k}\right) x\left(t_{k}\right)+g\left(t_{k+1}, t_{k}\right) \\
v^{o b s}\left(t_{k}\right)=\mathbf{C} x\left(t_{k}\right)+n\left(t_{k}\right)
\end{array}\right.
$$

with initial state $x_{0}$ as specified; $\left\{g\left(t_{k+1}, t_{k}\right)\right\},\left\{n\left(t_{k}\right)\right\}$, and $x_{0}$ are mutually independent.

As an alternative to the above approach resulting in the EDS as the model for the LDA velocity measurements, we will next consider an approach based on the following factorization of the known sampling instants

$$
t_{k}=k \Delta t+\tilde{t}_{k}=\tau_{k}+\tilde{t}_{k}
$$

with $\tau_{k}$, the $k$ th mean sampling instant and $\tilde{t}_{k}$ a deviation upon it. The Taylor series of observation process $\left\{v\left(t_{k}\right)\right\}$ developed about $\tau_{k}$ and truncated at, say, the $p$ th order time derivative is a linear approximation in terms of the 0th up to and including the $p$ th order time derivative of process $\{v(t)\}$. Now, the $p$ th order time derivative of process $\{v(t)\}$ depends upon the state process and the 0th up to and including the $(p-1)$ th order time derivative of process $\{h(t)\}$ with coefficients $\mathbf{C B}, \cdots, \mathbf{C A}^{(p-1)} \mathbf{B}$ respectively. Straightforward matrix manipulation shows that these coefficients disappear for $p \leq(n-m)$. This means that the expression for the $p$ th order time derivative of $\{v(t)\}$ simplifies considerably provided $p \leq \rho=(n-m)$. In view of these findings, the following model for the observation process is proposed

$$
\begin{aligned}
v^{o b s}\left(t_{k}\right) & =\mathbf{C} \sum_{p=0}^{\rho} \frac{\tilde{t}_{k}^{(p)}}{p} \mathbf{A}^{(p)} x\left(\tau_{k}\right)+n\left(\tau_{k}\right) \\
& =\tilde{\mathbf{C}}\left(\tilde{t}_{k}\right) x\left(\tau_{k}\right)+n\left(\tau_{k}\right)
\end{aligned}
$$


with $\left\{n\left(\tau_{k}\right)\right\}$ a zero mean discrete-time Gaussian white noise process with standard deviation $\sigma_{n}$. The alternative model for the fluid particle velocity measurements is incomplete without a propagation equation for evenly spaced state process $\left\{x\left(\tau_{k}\right)\right\}$. Fortunately, such an equation is readily obtained from the differential equation for $\{x(t)\}$. Consequently, the dynamic model for the observation process known as the approximately equivalent discrete-time system (AEDS) for the LDA velocity measurements is defined as

$$
\left\{\begin{array}{l}
x\left(\tau_{k+1}\right)=\Phi(\Delta t) x\left(\tau_{k}\right)+g\left(\tau_{k+1}, \tau_{k}\right) \\
v^{o b s}\left(t_{k}\right)=\tilde{\mathbf{C}}\left(\tilde{t}_{k}\right) x\left(\tau_{k}\right)+n\left(\tau_{k}\right) .
\end{array}\right.
$$

The mean and variance of process $\left\{g\left(\tau_{k+1}, \tau_{k}\right)\right.$ are evaluated as

$$
\begin{aligned}
\mathbf{E}\left\{g\left(\tau_{k+1}, \tau_{k}\right)\right\} & =\left\{\int_{0}^{\Delta t} e^{\mathbf{A} u} \mathbf{B} d u\right\} \mu \\
\mathcal{R}_{g}\left(\tau_{k}, \tau_{k}\right) & =\sigma_{h}^{2} \int_{0}^{\Delta t} e^{\mathbf{A} w} \mathbf{B B}^{T} e^{\mathbf{A}^{T} w} d w .
\end{aligned}
$$

Furthermore, $\left\{g\left(\tau_{k+1}, \tau_{k}\right)\right\},\left\{n\left(\tau_{k}\right)\right\}$, and $x_{0}$ are mutually independent. Seeing that the EDS as well as the AEDS requires advance knowledge of the sampling instants, any data processing based on one of these models is to be performed a posteriori.

\section{Estimating the Power SPectrum}

The LDA velocity measurements are known to be contaminated by random disturbances which affect any spectral estimate derived from these data. One way of dealing with additive stochastic measurement noise is to use a Kalman Filter [1] for noise compensation. For the design of this filter we require a describing stochastic dynamic model in the discretetime domain, i.e., either the EDS or the AEDS. As there is a choice between discrete-time models, there is choice between Kalman Filters: the equivalent discrete-time Kalman filter (EDKF) designed for the EDS, or the approximately equivalent discrete-time Kalman filter (AEDKF) designed for the AEDS. As for choosing between the two, the EDKF is, theoretically, preferable but the AEDKF yields an evenly spaced state estimator and is easier to implement.

It is a fact that the LDA turbulent velocity measurements are unevenly spaced in time. The best known and numerically most efficient spectral estimates use the FFT which demands evenly spaced measurements. This means that these estimation schemes can only be used in combination with the AEDKF, as the state process of the AEDS is evenly spaced. However, the applicability of the FFT-based spectral estimation schemes may be widened by adjusting the unevenly spaced measurements either through resampling or through estimation.

The resampling procedure breaks, roughly, down into two phases. In phase 1, a continuous-time process is reconstructed from the unevenly spaced discrete-time process. The most obvious reconstruction technique is the polynomial interpolation technique. It yields, in general, a continuous-time process $\{f(t)\}$ coincident with discrete-time process $\left\{f\left(t_{k}\right)\right\}$ at the individual sampling instants. In phase 2 , the reconstructed continuous-time process is sampled with some fixed sampling period.

Of the polynomial interpolation techniques employed, the step and linear interpolation techniques are the ones most frequently used. According to them, process $\{f(t)\}$ is

$$
\begin{aligned}
f_{\text {step }}(t)= & f\left(t_{m}\right) ; \\
f_{\text {lin }}(t)= & f\left(t_{m}\right)+\left(t-t_{m}\right) \frac{f\left(t_{m+1}\right)-f\left(t_{m}\right)}{t_{m+1}-t_{m}} \\
& \cdot t \in\left[t_{m}, t_{m+1}\right) .
\end{aligned}
$$

Although generalization to higher order interpolation schemes is obvious, it should be realized that such high order schemes do not necessarily yield better results. The reason is that when high order polynomials are fitted to randomly positioned points, periodic sampling can result in measurements well out of their original range. Although the polynomial interpolation technique is intuitively attractive and easy to implement, there is one drawback. It is namely possible to prove by means of a counter example that step interpolation, and therefore interpolation in general, applied to an unevenly spaced stationary discrete-time process results in a nonstationary continuoustime process. Consequently, the theoretical justification for using polynomial interpolation and resampling for the purpose of spectral estimation is thin.

The problem of adjusting the unevenly spaced observation process has, until now, been treated as an algebraic manipulation problem. There may, however, be a way in which to exploit the dynamic nature of the ESLM in order to obtain an evenly spaced observation process. Suppose $\tau_{k}=k \Delta t_{1}$ is a sequence of evenly spaced sampling instants and assume, for the sake of argument, that $m$ and $p$ are such that $t_{m} \leq$ $\tau_{p} \ldots \ldots<\tau_{p+q}<t_{m+1}$. It is not difficult to verify by means of the analytical solution of the ESLM, that for process $\left\{v\left(\tau_{p+j}\right)\right\}$ we find

$$
\begin{aligned}
v\left(\tau_{p+j}\right)=\mathbf{C} \Phi & \left(\tau_{p+j}-t_{m}\right) x\left(t_{m}\right) \\
& +\mathbf{C} g\left(\tau_{p+j}, t_{m}\right) ; \quad j \leq q
\end{aligned}
$$

with $\left\{g\left(\tau_{p+j}, t_{m}\right)\right\}$ a Gaussian discrete-time noise process for which

$$
\begin{aligned}
\mathbf{E}\left\{g\left(\tau_{p}, t_{m}\right)\right\} & =\left\{\int_{0}^{\tau_{p}-t_{m}} e^{\mathbf{A} u} \mathbf{B} d u\right\} \mu \\
\mathcal{R}_{g}\left(\tau_{p}, \tau_{p}\right) & =\sigma_{h}^{2} \int_{0}^{\tau_{p}-t_{m}} e^{\mathbf{A} w} \mathbf{B B}^{T} e^{\mathbf{A}^{T} w} d w
\end{aligned}
$$

In other words, the evenly spaced observation process $\left\{v\left(\tau_{p+j}\right)\right\}$ is a known function of discrete-time process $\left\{x\left(t_{m}\right)\right\}$. In practical situations, knowledge of state process $\left\{x\left(t_{m}\right)\right\}$ is almost always lacking. What is available though is its optimal estimator as produced by EDKF. This means, that by replacing process $\left\{x\left(t_{m}\right)\right\}$ with its optimal estimator we obtain an (optimal) estimator for evenly spaced particle velocity process $\left\{v\left(\tau_{j}\right)\right\}$. 


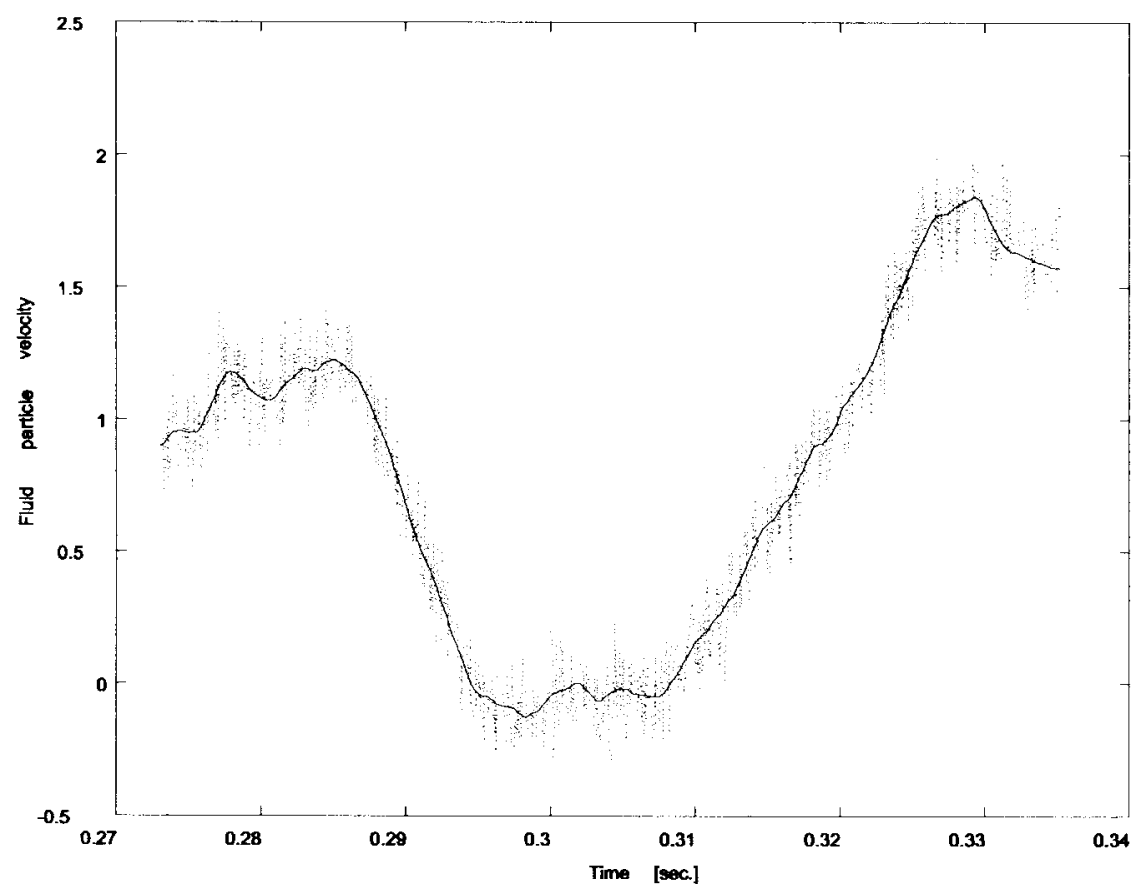

Fig. 1. Time history of the particle velocity process.

\section{EXAMPLE}

Although the development of the analysis methods reported in this paper was instigated by the problems encountered whilst estimating spectra on the basis of LDA measurement data, they are in fact applicable to a wide range of situations where Poisson sampling schemes are prevalent. For the purpose of demonstrating the analysis methods, consider the purely hypothetical ESLM determined by

$$
\begin{aligned}
& \mathbf{A}=\left(\begin{array}{c|cc}
-0.286 & 422 & 1.18 \times 10^{4} \\
\hline 0 & 0 & 1 \\
0 & -1.18 \times 10^{4} & -217
\end{array}\right) ; \\
& \mathbf{B}=\left(\begin{array}{c}
0 \\
0 \\
0 \\
1
\end{array}\right) ; \\
& \mathbf{C}=\left(\begin{array}{l|ll}
1 & 0 & 0
\end{array}\right) .
\end{aligned}
$$

The Gaussian white noise process $\{h(t)\}$ is completely determined by its mean $\mu=0.5$ and its standard deviation $\sigma_{h}=0.3$. Furthermore, the mean and variance of the Gaussian distributed initial state are selected in accordance with the specifications of Section II. Throughout this example Welch's Averaged Periodogram Method in combination with a Hanning Window, [5], is used for the actual power spectral density estimation. In accordance with this method, the data is divided into blocks of size $N_{F F T}\left(=2^{14}\right)$ with an overlap of $N_{\text {over }}(=$ $\left.2^{13}\right)$ points.

For the purpose of generating a time series of Poisson sampled measurements, a sequence of $N=2^{18}$ Poisson distributed sampling instants with mean sampling interval $\Delta t=2^{-14}$ sec is created. On the basis of the known sampling instants two time series of measurements are generated with the EDS; one consists of noise free measurements, while the other consists of noisy measurements, i.e., measurements affected by zero mean Gaussian white noise with standard deviation $\sigma_{n}=0.1$. A close-up view of the time history of these two time-series is available in Fig. 1; the solid graph represents the noise free measurements.

In this paper, several analysis methods for the continuoustime spectral estimation problem have been outlined. Before they are tested on the simulated noisy data, they are applied to the simulated noise free measurements. In Fig. 2 three such estimates (scaled down with $\Delta t$ ) derived from the noise free measurement sequence are displayed against $\mathcal{S}_{v}(\omega)$. Traces 1 and 3 have been derived from data adjusted by means of step and linear interpolation respectively before they were resampled at $\Delta t$. Application of the estimation adjustment technique for $\Delta t_{1}=\Delta t$ resulted in the spectral estimate represented by trace 2 .

It would appear that trace no. 3 represents the best estimate as it seems to lie closest to $\mathcal{S}_{v}(\omega)$. However, trace 2 is known to represent the best estimate the FFT algorithm can provide (used data is noise free), and it should therefore be the "de facto" benchmark for any other spectral estimate, and not $\mathcal{S}_{v}(\omega)$. Compared to trace 2, traces 1 and 3 display unknown aberrations resulting from the employed adjustment schemes.

The spectral estimates derived from the simulated noisy measurements are available in Fig. 3. Trace 1 is the nonscaled estimate determined after data adjustment through linear interpolation and resampling at $\Delta t$; trace 3 represents the scaled estimate obtained through application of aforementioned data adjustment scheme to the EDKF-filtered data. For scaled trace 2, the EDKF-filtered data has been adjusted by means of 


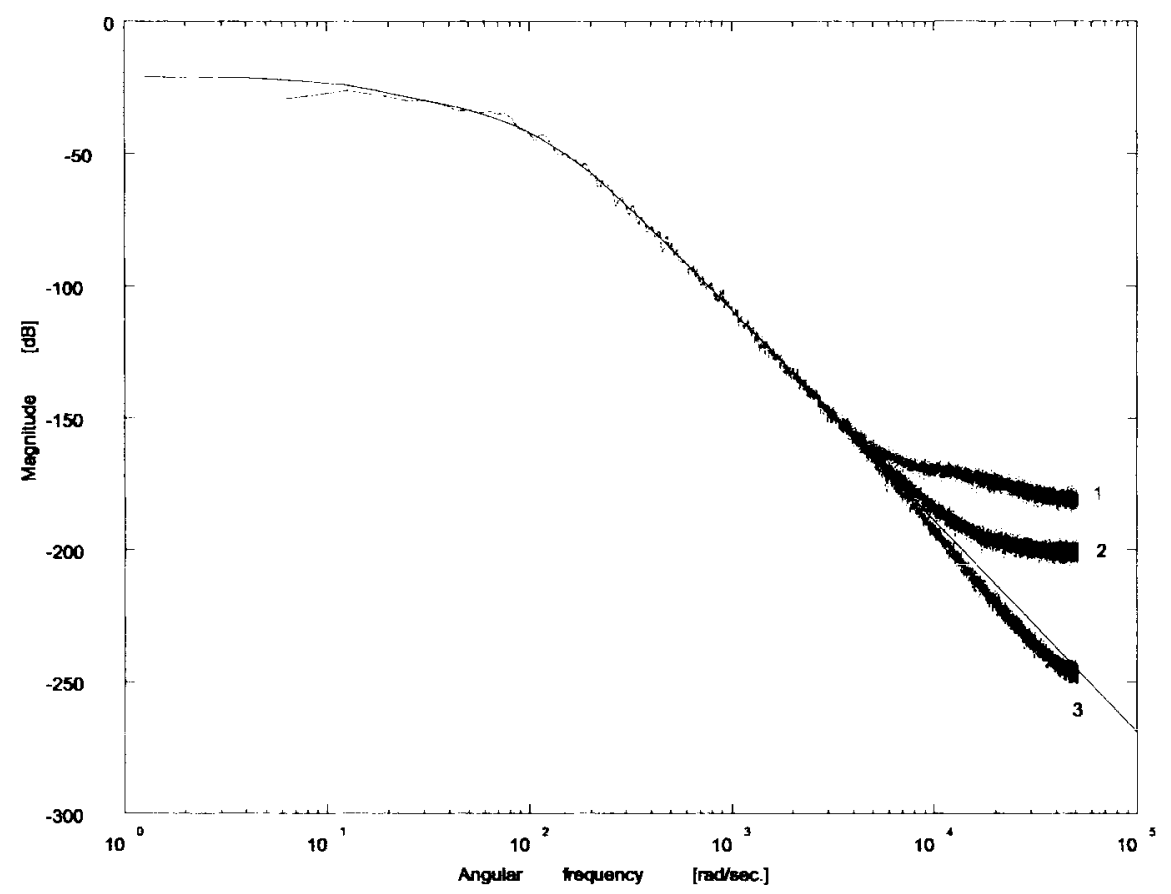

Fig. 2. Power spectral estimates.

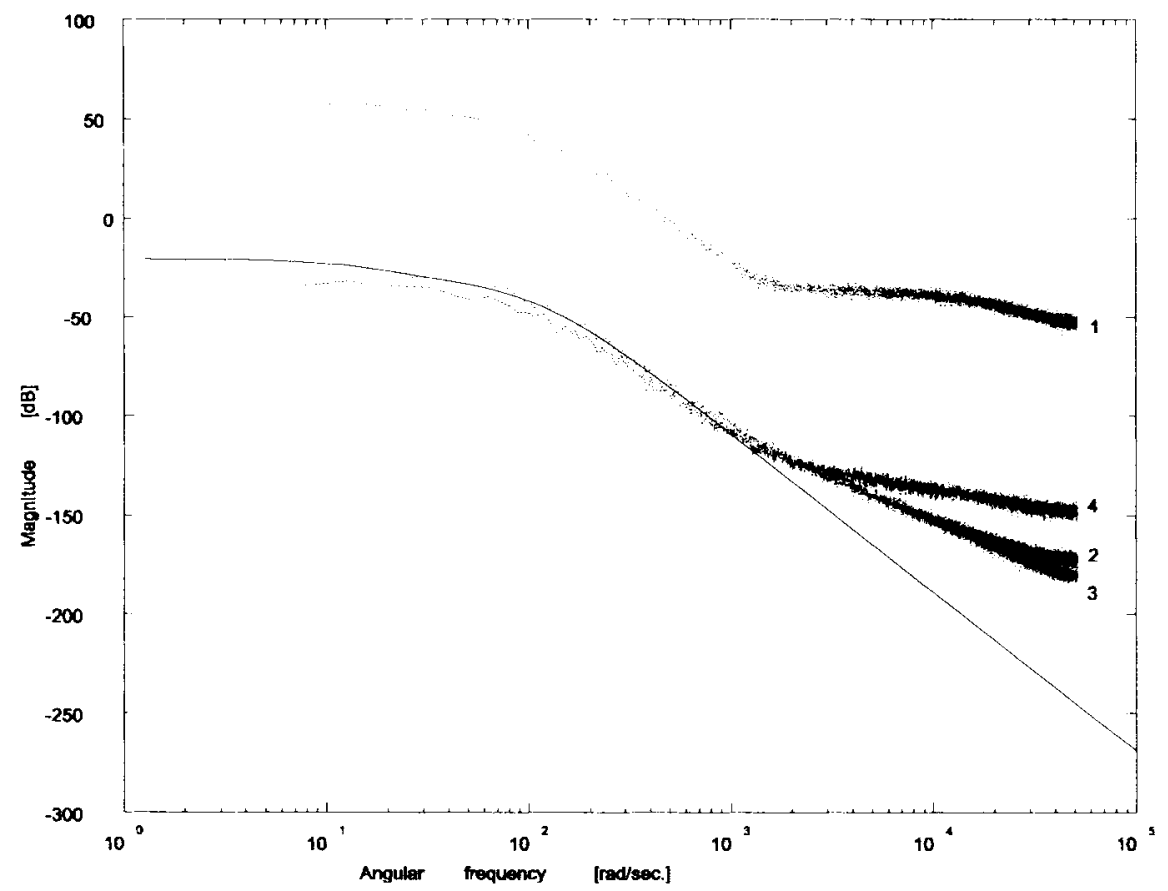

Fig. 3. Power spectral estimates.

estimation $\Delta t_{1}=\Delta t$ ). Trace 4 , also scaled, represents the spectral estimate directly derived from AEDKF- filtered data.

\section{CONCLUSION}

In this paper, the problems associated with obtaining spectral estimations from LDA data have served as a vehicle for the development of spectral analysis methods suitable for Poisson sampled measurements in general. In the course of the analysis both an exact and an approximate model have been developed for such measurements. The approximate character of the latter model was the price to be paid in return for an evenly spaced state process. For both digital models the Kalman Filter exist, and can be used to reduce measurement noise levels.

The paper discusses four different FFT-based spectral estimation schemes of which two have been newly developed. Theoretical considerations supported by simulation results 
indicate that the novel idea of adjusting the unevenly spaced measurements via estimation using the Kalman Filter yields the preferred method of analysis. Although the Kalman Filter for the approximate model produces an evenly spaced state estimator, the simulations show an inferior reliability due to as yet unexplained numeric sensitivity.

\section{REFERENCES}

[1] B. D. O. Anderson and J. B. Moore, Optimal Filtering. Englewood Cliffs, NJ: Prentice-Hall, 1979.

[2] S. B. Pope, "Lagrangian PDF methods for turbulent flows," Ann. Rev. Fluid Mech., vol. 26, 1994.

[3] A. E. Perry, K. L. Lim, and S. M. Henbest, "An experimental study of the turbulence structure in smooth- and rough-wall boundary layers," $J$. Fluid Mech., vol. 177, pp. 437-466, 1987.

[4] E. Masry, D. Klamer, and C. Mirabile, "Spectral estimation of continuous-time processes: Performance comparison between periodic and Poisson sampling schemes," IEEE Trans. Automat. Contr., vol. AC-23, pp. 679-685, Aug. 1978.

[5] A. V. Oppenheim and R. W. Schafer, Digital Signal Processing. Englewood Cliffs, NJ: Prentice-Hall, 1975.

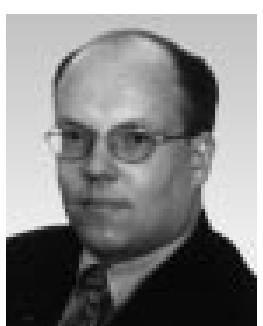

Reinder Banning received the M.Sc. degree from the Faculty of Applied Mathematics, the University of Twente, The Netherlands, in 1989 and the Ph.D. degree from the University of Strathclyde, Glasgow, Scotland, in 1993.

Since then, he has been with the Faculty of Applied Physics, the Delft University of Technology, Delft, The Netherlands, as a Lecturer in the field of systems and control. 